8- 12 February $\quad \mathbf{1 7}^{\text {th }}$ International Symposium on Microscale Separations and Analysis (HPCE 2004), Salzburg, Austria. Chairman Prof. Wolfgang Lindner. Information: Congress Secretariat, Mrs Ina Kaehler, PCO Tyrol Congress, Rennweg 3, 6010 Innsbruck, Austria. Tel. +43 (512) 575600; Fax +43 (512) 575607.

E-Mail: c.kaehler@congress-innsbruck.at;www.hpce2004.at

2-6 May $\mathbf{1 5}^{\text {th }}$ International Symposium on Pharmaceutical and Biomedical Analysis, Florence, Italy. Information Dr. Sergio Pinzauti, Chairman, University of Florence, Via G. Capponi 9, 50121 Florence, Italy.

Tel.+39055 275 7284; Fax +39055240 776. E-Mail: info@pba.com

5-8 May

XIth International Symposium on Luminescende Spectrometry in Biomedical and Environmental Analysis Spectrosopic and Imaging Detection Techniques, Tsinghua University, Beijing, China. Information: Professor Dr. Xinrong Zhang, Analysis Center, Department of Chemistry, Tsinghua University, Beijing 100084, P. R. China. Tel. +86 (10) 62781688 (Lab); Fax +86 (10) 62770327 (Lab). E-Mail: xrzhang@chem.tsinghua.edu.cn

\title{
Erratum
}

\section{Properties of Reversed Phase Packings with an Embedded Polar Group}

by U. D. Neue* / Y.-F. Cheng / Z. Lu / B. A. Alden / P. C. Iraneta / C. H. Phoebe / K. Van Tran

published in 2001, 54, 169-177

Figure 11 on page 176 was incorrect. The correct version of this figure is shown here.

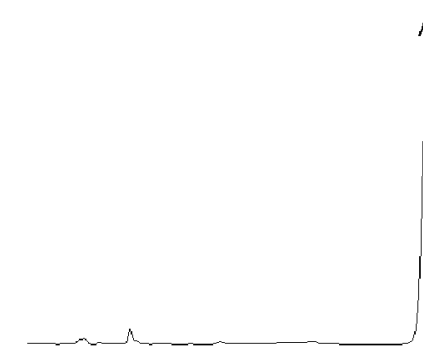

A

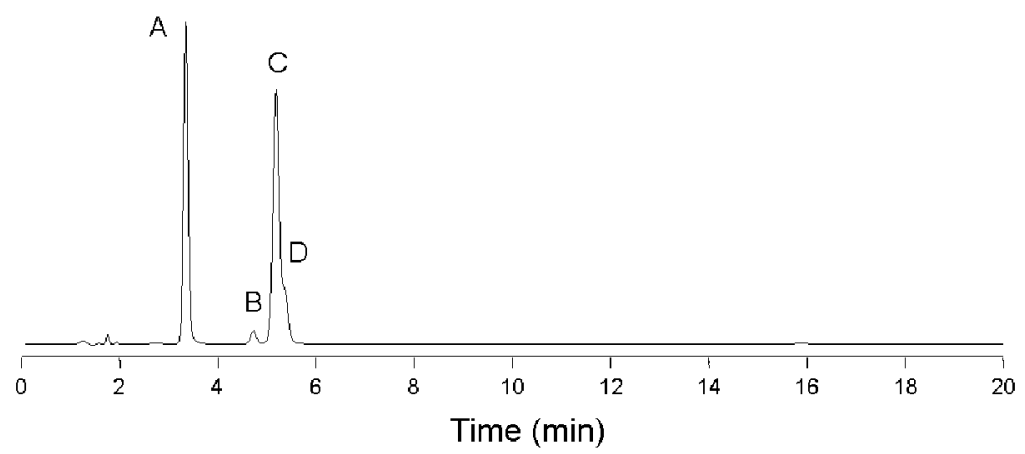

Figure 11. Separation of quercetin (A), kaempferol (C) and isorhamnetin (D). Peak B is a contamination of kaempferol. Columns: SymmetryShield RP 8 (top) and $\mathrm{SymmetryC}_{8}$ (bottom), $4.6 \times 150 \mathrm{~mm}, 5 \mu \mathrm{m}$. Mobile phase $55 \%$ water, $35 \%$ acetonitrile, $10 \% 50 \mathrm{mM}$ formic acid. Flow rate: $1.2 \mathrm{~mL}$ min ${ }^{1}$. Column temperature: $35^{\circ} \mathrm{C}$. Detection: UV at $270 \mathrm{~nm}$. 